\title{
New Technique for Evaluation of Preferential Alignment of Biological Apatite (BAp) Crystallites in Bone Using Transmission X-ray Diffractometry
}

\author{
Katsunari Sasaki ${ }^{1,2, * 1}$, Takayoshi Nakano ${ }^{3, * 2}$, Joseph D. Ferrara ${ }^{2}$, \\ Jee-Wook Lee ${ }^{3}$ and Toshihiko Sasaki ${ }^{1}$ \\ ${ }^{1}$ Division of Innovative Technology and Science, Graduate School of Natural Science \& Technology, \\ Kanazawa University, Kanazawa 920-1192, Japan \\ ${ }^{2}$ Rigaku Corporation, Akishima 196-8666, Japan \\ ${ }^{3}$ Division of Materials and Manufacturing Science, Graduate School of Engineering, Osaka University, Suita 565-0871, Japan
}

Two-dimensional quantitative analysis of point focus X-ray beam diffraction (XRD) was performed using a transmission optics system to examine the biological apatite (BAp) orientation in the femurs of an osteopetrotic (op/op) mouse and a normal mouse. The Mo K $\alpha$ (wavelength: $0.07107 \mathrm{~nm}$ ) was used as characteristic X-ray radiation as a substitute for conventional $\mathrm{Cu} \mathrm{K} \alpha$ (wavelength: $0.15418 \mathrm{~nm}$ ) radiation. At first a theoretical calculation concerning with X-ray absorption and peak resolution was performed, and subsequently X-ray diffraction measurements were carried out to confirm the usefulness of the transmission X-ray method by Mo K $\alpha$ radiation. The distribution of the preferential orientation of the BAp $c$-axis was finally measured and calculated as an integrated intensity ratio of $(002) /(310)$ in a plane roughly containing the bone longitudinal axis. The result resembled analysis from a conventional reflection X-ray diffraction method by $\mathrm{Cu} K \alpha$ radiation but this transmission optics system was more convenient for the screening of BAp orientation in bones without sectioning.

The distribution of the preferential alignment of the BAp $c$-axis was measured as a function of the longitudinal axis in the femurs of a 12 week mutant osteopetrotic (op/op) mouse and a littermate control mouse. The preferential alignment of the BAp $c$-axis along the longitudinal direction is much lower in the op/op mouse than in the control mouse in all analytical positions. The transmission optics system using Mo K $\alpha$ radiation in this study provides a fuss-free method for analyzing BAp orientation as a bone quality parameter.

[doi:10.2320/matertrans.MER2008160]

(Received May 19, 2008; Accepted June 16, 2008; Published August 25, 2008)

Keywords: op/op, osteopetrosis, biological apatite crystallite, bone quality, $X$-ray diffraction, transmission method, X-ray absorption, imaging plate

\section{Introduction}

X-ray diffraction is a well-known, useful method to evaluate materials properties. Diffraction is caused by reflection of the X-ray on the crystal plane, so this method is mainly used to evaluate the crystal properties such as lattice parameters, crystallinity, preferred orientation, residual stress, etc. In general, crystalline structural materials based on metals, ceramics, and plastics are originally composed of randomly or weakly oriented crystallites. Modification of the crystal property such as preferred orientation and residual stress, however, can control or improve the materials properties. Recently, the X-ray diffraction technique has been dramatically improved by the development of such two-dimensional detectors as Imaging Plate (IP), Multi Wire Proportional Counter (MWPC), and Charge Coupled Device (CCD). ${ }^{1)}$ These techniques provide two-dimensional information on the basis of the crystal properties in the materials, dramatically reducing measurement time. ${ }^{2)}$

Bone is a typical biological structural material that requires mechanical properties to support body weight and to protect the internal organs. Bone tissue is a composite dominantly comprised of biological apatite (BAp) crystallite as its mineral constituent and collagen as its organic matrix. ${ }^{3)}$ The mineral component of bone tissue has two primary functions: mechanical and homeostatic. The BAp crystals reinforce the organic matrix by giving it strength and rigidity, while also serving as a repository of $\mathrm{Ca}, \mathrm{Mg}$, inorganic phosphate

\footnotetext{
${ }^{* 1}$ Graduate Student, Kanazawa University

${ }^{* 2}$ Corresponding author, E-mail: nakano@mat.eng.osaka-u.ac.jp
}

ions, etc. To perform their crucial role in bone, BAp crystallites have a relatively narrow range of sizes $(\sim 1000 \mathrm{~nm})$, and generally their mineral properties differ depending on age, health, or the presence of disease. ${ }^{4)}$ Understanding the structural and chemical transformations of BAp and the role of environmental constraints in determining the structure and the composition of ultimate bone mineral are all relevant biological and medical issues. ${ }^{5)}$

Bone is usually evaluated using bone mineral density (BMD). But recent research suggests that BMD is an insufficient predictor of bone mechanical properties and is related to bone strength and fracture risk. ${ }^{6,7)}$ In the various parameters of bone microstructure, Nakano et al. have been focusing on the preferential orientation of BAp crystallites analyzed by a micro-beam X-ray diffraction technique because the preferential alignment of the BAp $c$-axis in various bones varies depending on the shape, the stress distribution in vivo, and the related mechanical function. ${ }^{8)}$ The analysis of BAp orientation also clarifies the bone regeneration process under a tissue engineering technique since BAp orientation and BMD independently change during bone regeneration. ${ }^{9,10)}$

$\mathrm{Cu} \mathrm{K} \alpha$ radiation is used for almost all X-ray diffraction studies. Since the absorption coefficient of the X-ray beam is quite large through the BAp crystallite, the $\mathrm{Cu} K \alpha$ beam can only provide information about the bone surface, ${ }^{11)}$ so it is not sufficient for biomedical applications, even on isolated bone specimens; the specimen must be exposed by cutting when internal bone microstructure is analyzed.

Thus, the purpose of this study is to apply the transmission $\mathrm{X}$-ray diffraction method with higher energy characteristic 

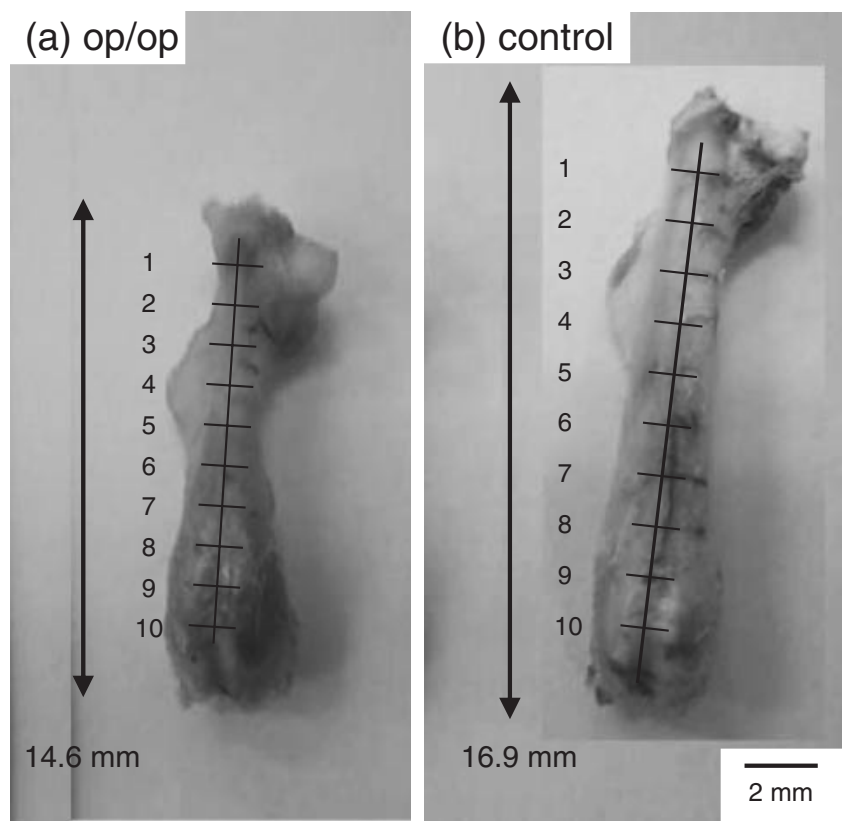

Fig. 1 Appearance of mouse femurs used and analyzed positions for X-ray diffractometry: (a) op/op; (b) control.

$\mathrm{X}$-ray Mo $\mathrm{K} \alpha$ radiation on mouse femur without sectioning. As a result, high energy radiation reduced the effect of absorption due to improvements of the signal-to-noise ratio and successfully analyzed the internal microstructure in bone specimens without cutting. The distribution of the BAp $c$-axis was clarified as a function of longitudinal bone axis in the femur bone of a 12-week osteopetrotic (op/op) mouse in which osteoclasts rarely appear due to a defect in the expression of the macrophage colony-stimulating factor (M-CSF) compared with the littermate mouse as a control using a transmission X-ray diffraction system.

\section{Materials and Methods}

\subsection{Bone and reference specimens}

Two right femurs were removed from a 12-week-old op/op mouse and the littermate mouse, fixed, and kept in a $10 \%$ formalin neutral buffered solution. Figure 1 shows a photograph of the bones used for this experiment. The op/op mouse specimen is from a mutant osteopetrotic (op/op) mouse, and the control specimen is from a normal mouse, a littermate of the op/op mouse. Since the femur of op/op mouse is shorter than that of the control mouse, the op/op mouse showed typical osteopetrosis.

\subsection{X-ray micro-CT}

The internal structure of each bone was observed by a Rigaku R_mCT equipped with a micro X-ray CT scanner especially designed for small animal imaging. The unit operated X-ray power at $90 \mathrm{kV}$ and $88 \mu \mathrm{A}$ with an exposure time of $17 \mathrm{~s}$.

\subsection{X-ray diffraction analysis}

All the X-ray diffraction experiments were performed using a prototype of the Rigaku R-AXIS Bone Quality (BQ) system. As the X-ray detector, this system employs an

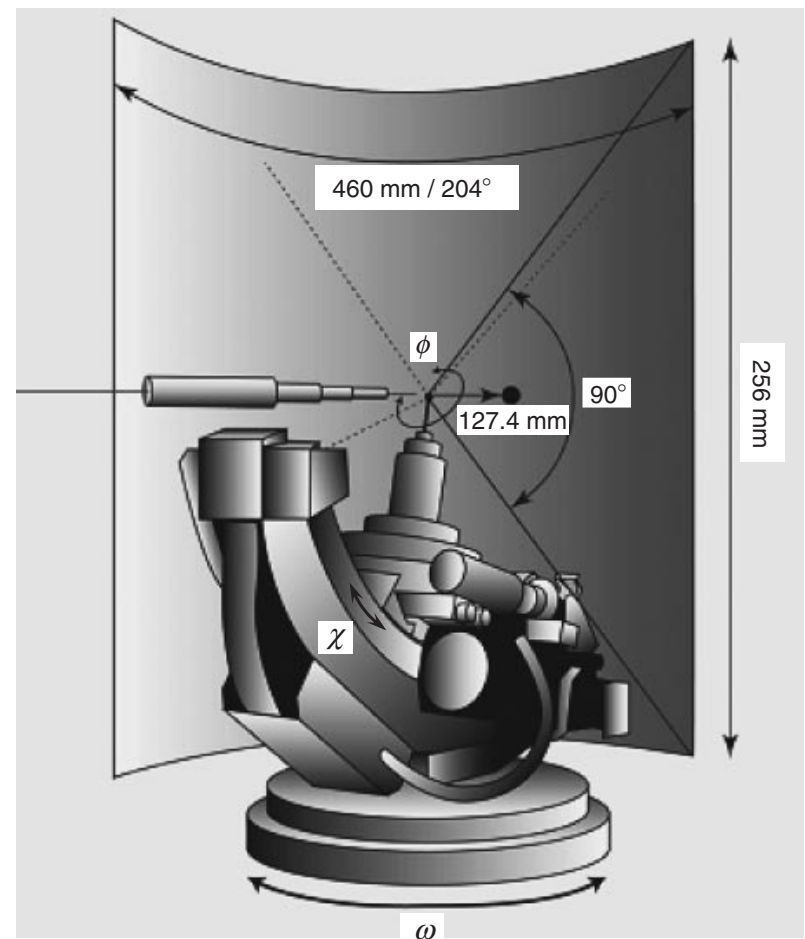

Fig. 2 Schematic drawing of Rigaku R-AXIS BQ goniometer prototype and $\mathrm{X}$-ray detecting area by imaging plate as a diffraction detector.

imaging plate (storage phosphors) developed by Fuji Film Co., Ltd. The geometry of the apparatus, which resembles a vertical Weissenberg camera, was mainly designed for single crystal X-ray structure analysis and charge density study. Figure 2 shows a schematic drawing of the prototype of the Rigaku R-AXIS BQ X-ray optics system. The distance between the detector and the X-ray focus of the specimen is $127.4 \mathrm{~mm}$ in a horizontal direction. The detected area on the imaging plate is $256 \mathrm{~mm} \times 460 \mathrm{~mm}$ with a curvature on the horizontal plane. This provides a $2 \theta$ range from $-60^{\circ}$ to $+144^{\circ}$ in the horizontal direction and a $2 \theta$ range of $\pm 45^{\circ}$ in the vertical direction. The pixel area of the imaging plate is $100 \mu \mathrm{m} \times 100 \mu \mathrm{m}$. In the study, a Rigaku ultra X rotating anode $\mathrm{X}$-ray generator with either a copper $(\lambda=0.15418$ $\mathrm{nm})$ or a molybdenum $(\lambda=0.07107 \mathrm{~nm})$ target was used accompanied with a graphite monochromator. The monochromator provides substantial monochromatic radiation of the wavelength. A $0.3-\mathrm{mm}$ double-pinhole metal collimator was used, and the X-ray generator was operated at $50 \mathrm{kV}$ and $90 \mathrm{~mA}(4.5 \mathrm{~kW})$. Both copper and molybdenum radiation were used to examine the behavior of the X-ray absorption in the NIST apatite (hydroxyapatite; NIST \#2910) powder which was inserted into a glass capillary (diameter: $1.5 \mathrm{~mm}$ ). The exposure times of the X-ray Mo K $\alpha$ radiation for the NIST apatite and the bone specimens were 10 minutes and 1 minute, respectively.

The specimens were oriented on a 3-circle Eulerian goniometer with an X-Y-Z stage. The Eulerian angles were $\phi, \chi$, and $\omega$. The $\phi$-axis was closest to the specimen and provided complete specimen rotation around that axis. The $\chi$-axis, the next closest to the specimen, was used to tilt the specimen. In general, the other $\omega$-axis is used as a scanning axis. The specimens were moved to their desired positions by 


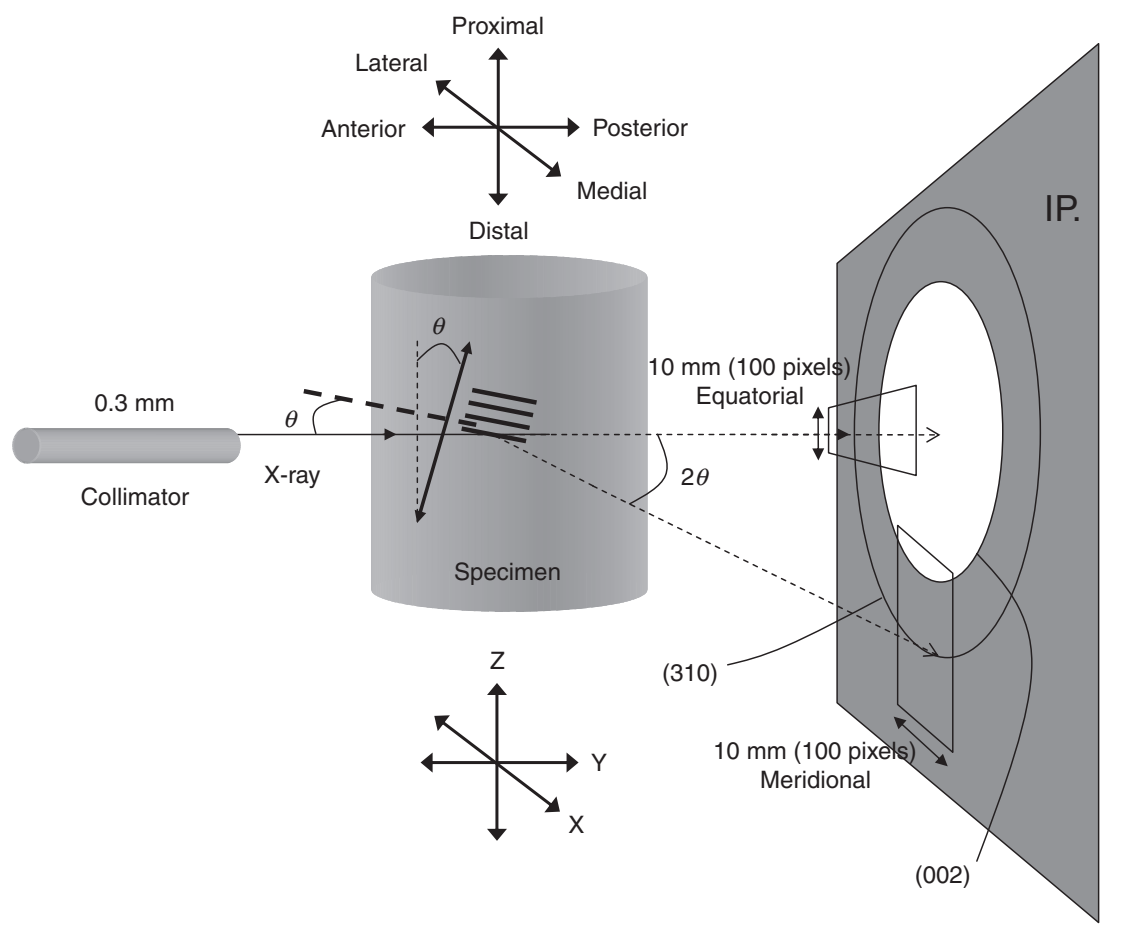

Fig. 3 Schematic drawing of experimental conditions: X-ray optics system, definition of each axis, and focused area on imaging plate as a diffraction detector.

the X-Y-Z stage. The distribution of the X-ray absorption of the bone specimens was measured by a Rigaku X-ray scintillation counter operating in the integrating mode. It is easy to change the transmitted X-ray direction against the specimen between the anterior-posterior and medial-lateral directions by rotation of $90^{\circ}$ around the $\phi$ axis.

Figure 3 shows a schematic drawing of the optics system and the obtainable diffraction rings. The X-ray diffraction data were recorded using Rigaku R-AXIS BQ software. For each bone specimen, measurements were carried out at the 10 positions described in Fig. 1. The measurements were repeated three times after removing and again fixing the specimen on the X-Y-Z stage. For directions parallel and perpendicular to the longitudinal bone axis, a $10-\mathrm{mm}$ length on the imaging plate along the tangential line to the diffraction rings was extracted in both the equatorial and meridional areas of the images, and the intensity of the diffracted beams in the corresponding area was converted as a function of $2 \theta$ as an X-ray diffraction profile. In the X-ray profile, the (002) and (310) reflections were identified, and the integrated intensity and the intensity ratio of (002) to (310) were calculated. The intensity ratio corresponds to the preferential alignment of the $c$-axis of the BAp crystallites, as shown previously. ${ }^{8}$ )

\section{Results and Discussion}

\subsection{Micro-CT images}

Figure 4 shows cross-sections based on the micro-CT images of the perpendicular and parallel directions to the longitudinal bone axis in the femurs of the op/op and control mice. Bone malformation was clearly found in the op/op mouse. The longitudinal axis of the femur of the op/op mouse was shorter than that in the control mouse. In arbitrary cross-sections perpendicular or parallel to the longitudinal direction in the femurs, the calcified area relative to the total cross-sectional area was greater in the op/op mouse than in the control mouse, which means that the op/op mouse shows typical osteopetrosis with superfluous bone formation. In other words, the femur of the op/op mouse possesses developed cancellous bone in the medullary cavity within the thin wall of the cortical bone, while bone trabeculae only appear in the metaphyseal portion of the femur in the control mouse.

\subsection{Absorption}

$\mathrm{X}$-ray absorption is described by the following equation:

$$
I=I_{0} e^{-\mu t},
$$

where $I$ is the intensity of the transmitted X-ray beam, $I_{0}$ is the intensity of the incident X-ray beam, $\mu$ is the absorption coefficient in $\mathrm{cm}^{-1}$, and $t$ is the thickness of the sample in $\mathrm{cm}$. In general, X-ray absorption quickly decreases with increasing wavelength. In the case of hydroxyapatite, a nominal density of $3.19 \mathrm{~g} / \mathrm{cm}^{2}, \mu=159.09 \mathrm{~cm}^{-1}$ for $\mathrm{Cu} \mathrm{K} \alpha$, and $\mu=19.12 \mathrm{~cm}^{-1}$ for Mo K $\alpha$ can be given. Substituting these values into the above equation with a thickness of $0.05 \mathrm{~cm}$ for the bone specimen indicates that $99.96 \%$ of the $\mathrm{Cu} \mathrm{K} \alpha$ $\mathrm{X}$-ray would be absorbed but only $38.4 \%$ of Mo $\mathrm{K} \alpha \mathrm{X}$-ray would be absorbed. This suggests that Mo K $\alpha$ X-ray radiation is a much better choice for this experiment for using a transmission optics system.

Confirmation of this claim is shown experimentally in Fig. 5, which shows diffraction patterns for NIST apatite using $\mathrm{Cu} \mathrm{K} \alpha \mathrm{X}$-ray and Mo $\mathrm{K} \alpha \mathrm{X}$-ray radiation. The only difference between the exposures is the radiation type; all 

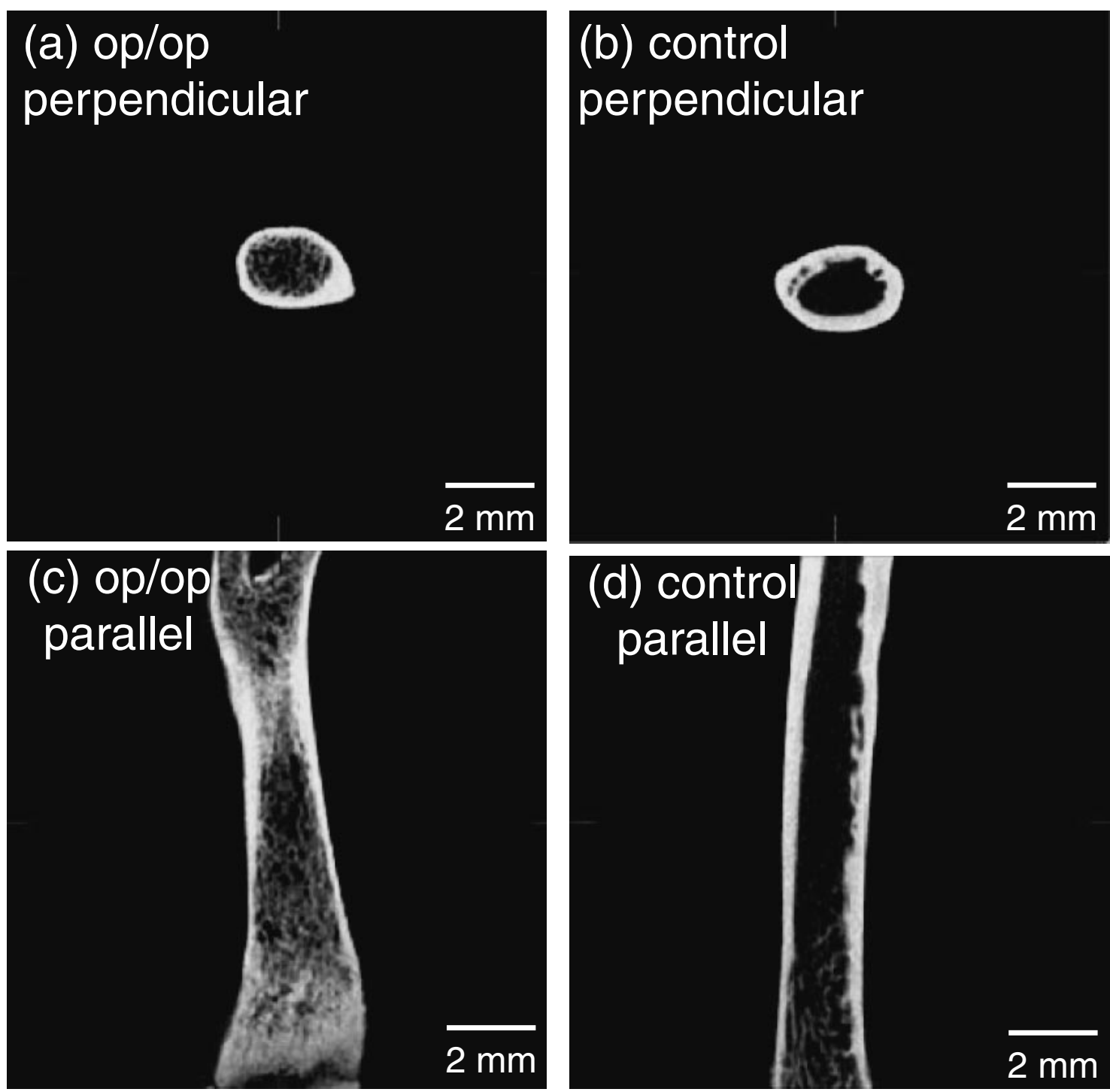

Fig. 4 X-ray micro-CT images perpendicular and parallel directions to longitudinal bone axis in femurs of op/op and control mice: (a) op/op mouse, perpendicular direction, (b) control mouse, perpendicular direction, (c) op/op mouse, parallel direction, (d) control mouse, parallel direction.

other experimental variables were held constant. The pattern exposed by the $\mathrm{Cu} \mathrm{K} \alpha \mathrm{X}$-ray is obviously of lower quality than that by the Mo K $\alpha$ X-ray.

An absorption profile of the two bones irradiated by Mo $\mathrm{K} \alpha \mathrm{X}$-ray radiation is shown in Fig. 6. The absorption profile reveals that the peak transmission is about $40 \%$ to $20 \%$, well within the range of reasonable values for transmission mode diffractometry. The curves show higher bone density at the edges for the control specimens due to the cortical portion and higher density in the center for the op/op specimen, which is consistent with the micro-CT images of the developed trabeculae, as shown in Fig. 4.

\subsection{Angular resolution and diffraction plane tilting}

The disadvantage of Mo $\mathrm{K} \alpha \mathrm{X}$-ray radiation is the reduced angular resolution of the reflections in the resultant data. Figure 7 shows a typical Mo K $\alpha$ X-ray diffraction pattern of the representative bone specimen. The present work compares the ratio of the integrated intensities of the (002) and (310) reflections. Additionally, Fig. 7 displays the enlarged diffraction pattern of the $10^{\circ} \leq 2 \theta \leq 20^{\circ}$ region. This indicates these reflections are well resolved with the Mo $\mathrm{K} \alpha$ $\mathrm{X}$-ray radiation due to the essentially low crystallinity of BAp in bones.

BAp crystallites orient preferentially along the longitudinal bone axis in long bones, and the geometry that optimizes the (002) and (310) reflections requires around a $6^{\circ}$ and $9^{\circ}$ tilt of the bone axis, respectively, for the $\mathrm{Mo} \mathrm{K} \alpha \mathrm{X}$-ray radiation. We collected a series of images from $-50^{\circ} \leq \chi \leq 50^{\circ}$ to determine the relationship of the $(002) /(310)$ ratio as a function of the tilt angle to the longitudinal bone axis. The preferred orientation of the $c$-axis is to the longitudinal axis of the bone, but the pole distribution is very broad around the longitudinal bone axis (data not shown). This suggests that tilting toward the bone axis under consideration of the Bragg angles is not necessary to get meaningful results. Thus, the deviation angle of the detected reflection plane, normal from the longitudinal bone axis, can be ignored even from the (310) plane, which deviates within $9^{\circ}$ due to the short wavelength of the Mo $\mathrm{K} \alpha \mathrm{X}$-ray radiation. 
(a) $\mathrm{Cu} \mathrm{K \alpha}$

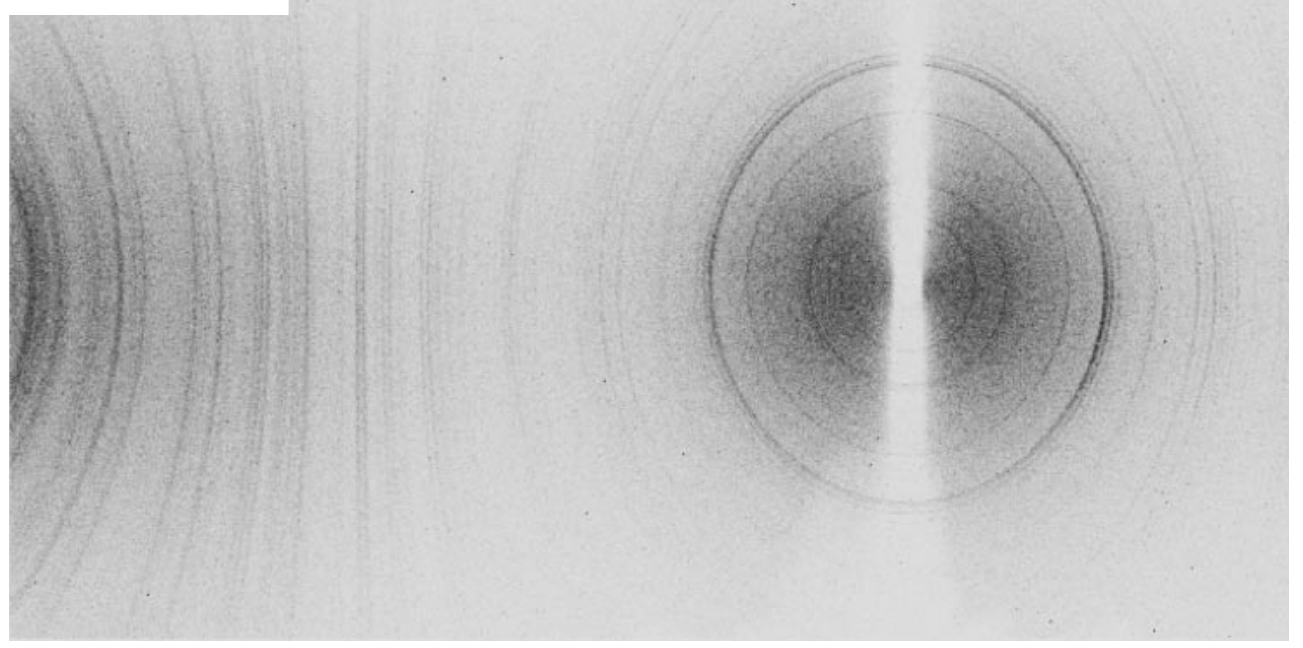

(b) Mo K $\alpha$

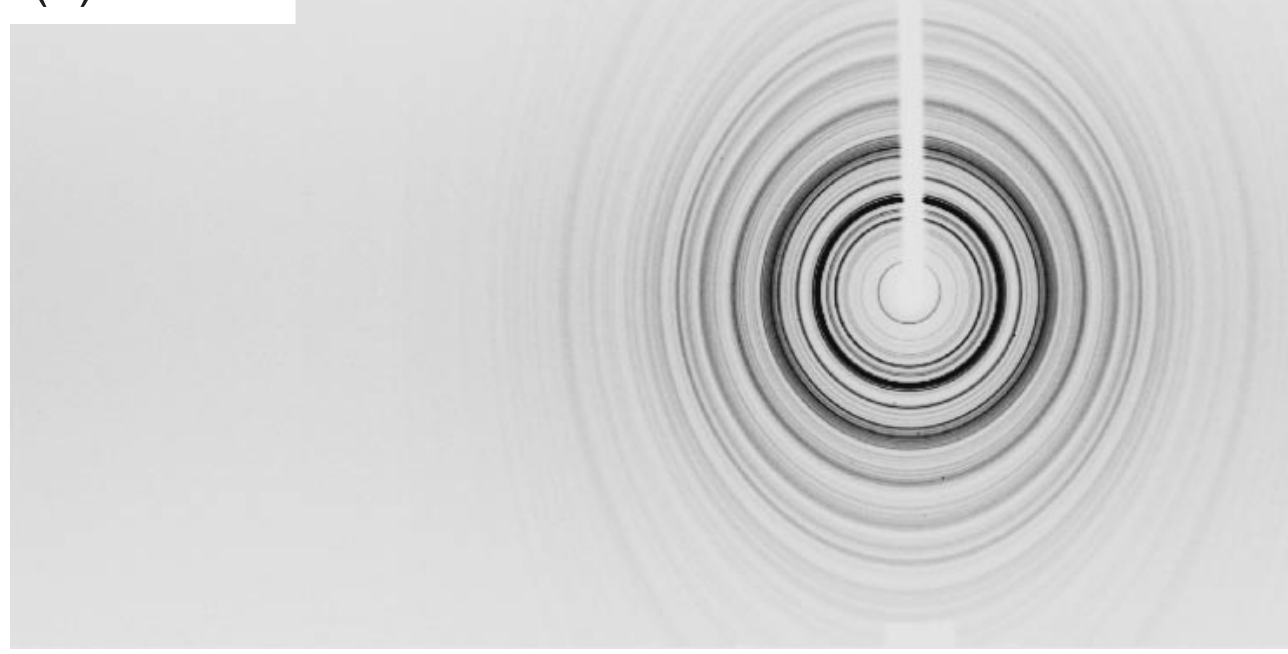

Fig. 5 X-ray diffraction patterns from NIST apatite powder placed in $1.5 \mathrm{~mm}$ diameter glass capillary. X-ray power is $50 \mathrm{kV}$ and $90 \mathrm{~mA}$ and exposure time is $10 \mathrm{~min}$ : (a) $\mathrm{Cu} \mathrm{K} \alpha \mathrm{X}$-ray radiation, (b) $\mathrm{Mo} \mathrm{K} \alpha \mathrm{X}$-ray radiation.

(a) lateral to medial direction

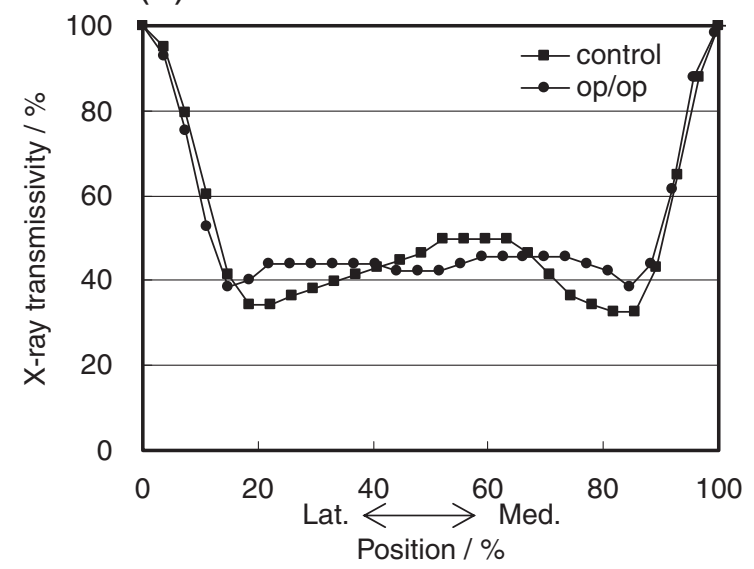

(b) anterior to posterior direction

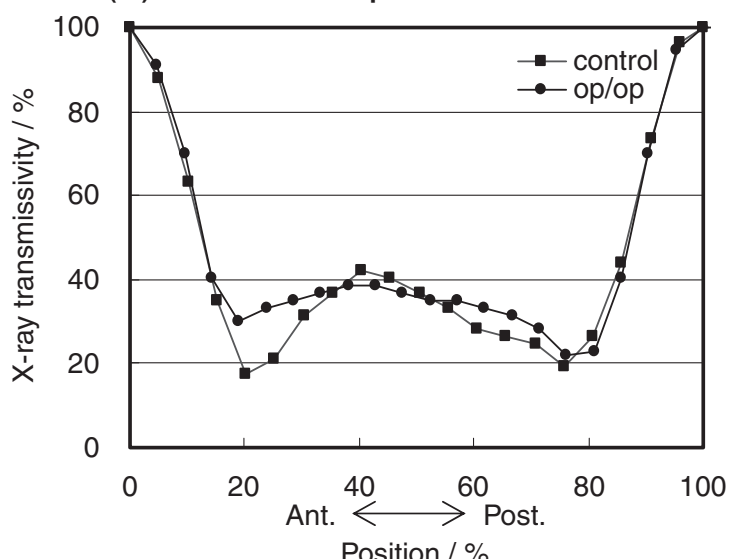

Fig. 6 Distribution of degree of X-ray transmissivity as a function of bone position to normalized whole bone width at Mo K $\alpha$ X-ray radiation: (a) lateral to medial direction, (b) anterior to posterior direction. 


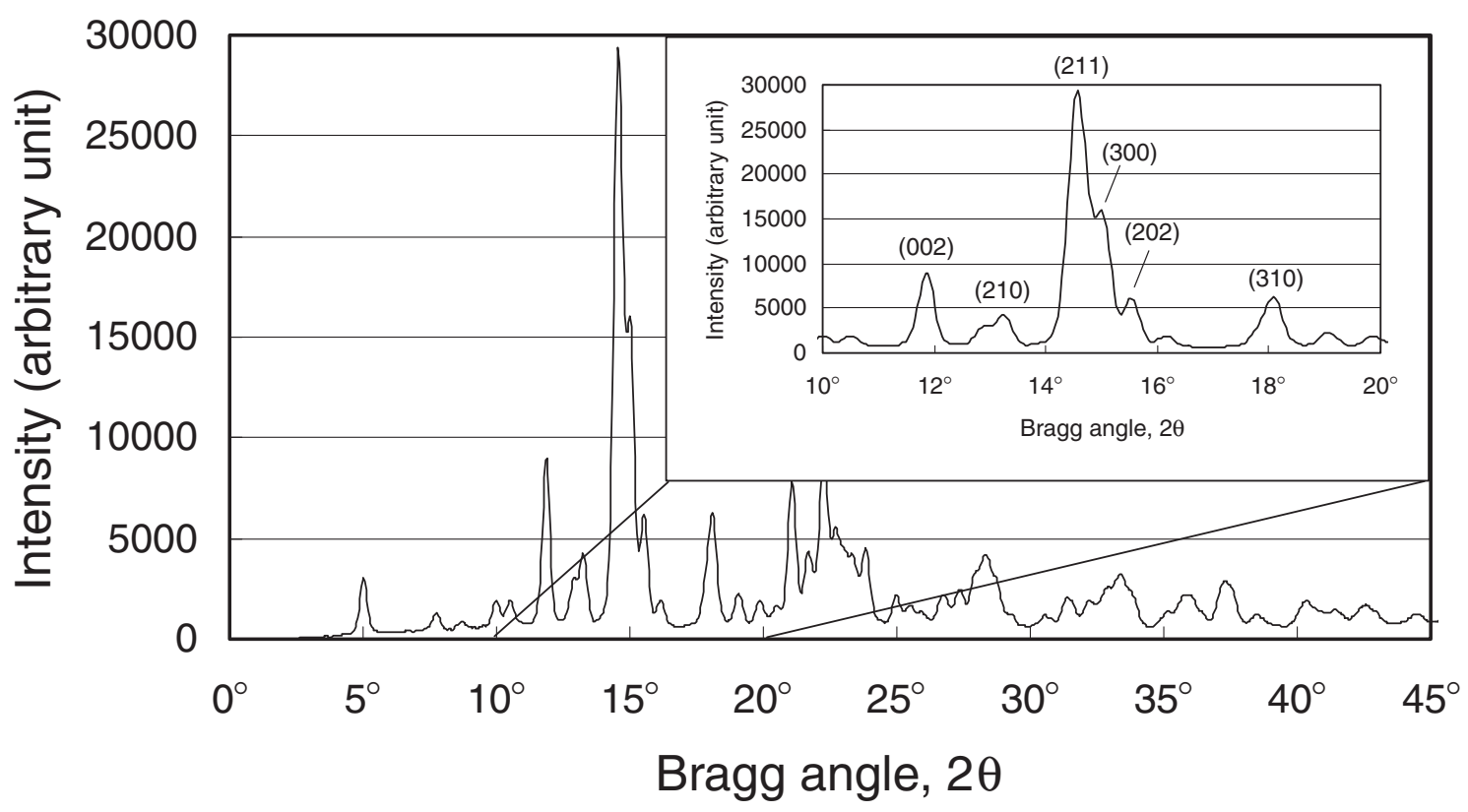

Fig. 7 Typical X-ray diffraction pattern of bone specimen taken by Mo K $\alpha$ X-ray radiation. Intensity in equatorial 100 pixels (10 mm) on equator (Fig. 3) accumulated and converted to $2 \theta$ versus intensity data.

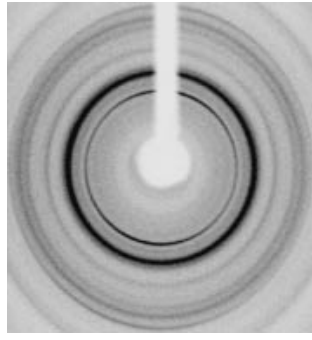

op/op: 1

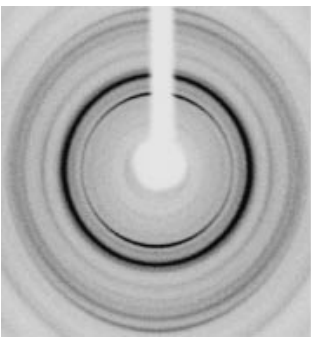

control: 1

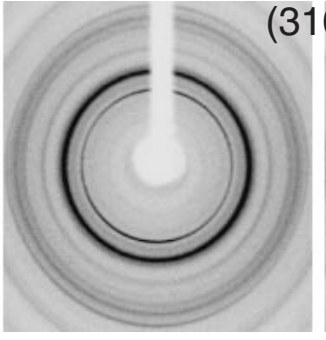

3

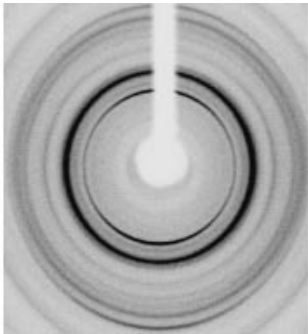

3

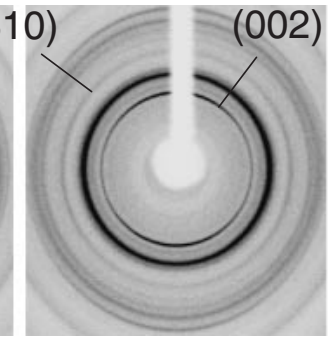

5

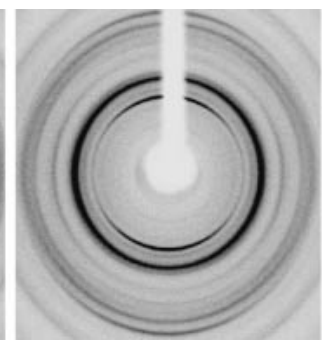

5

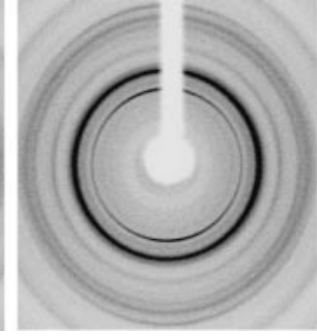

7

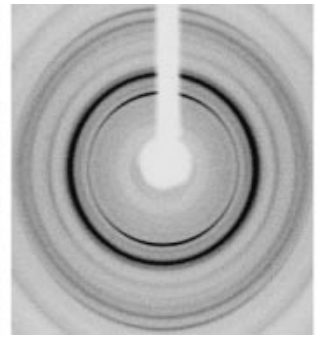

7

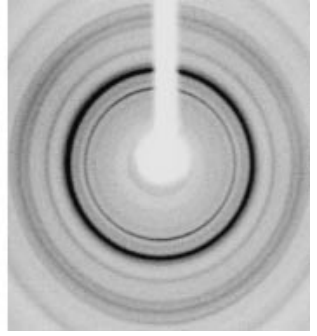

9

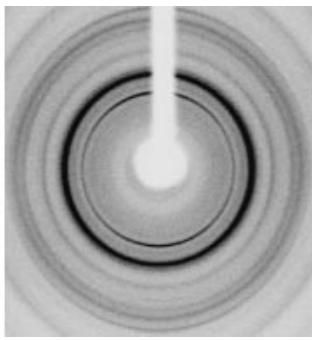

9

Fig. 8 X-ray diffraction patterns for specimen positions 1, 3, 5, 7, and 9; upper: op/op mouse, lower: control mouse. (002) and (310) diffraction rings were focused.

\subsection{Measurement of distribution of BAp orientation as a bone quality parameter}

Finally, we measured a series of 10 diffraction patterns at equidistant points along the longitudinal axis of the two types of bone specimens. The measurement points are shown in Fig. 1. The diffraction images at points 1, 3, 5, 7, and 9 for both the op/op and control mice bones are shown in Fig. 8. Each diffraction pattern was analyzed as described earlier, and the resultant $(002) /(310)$ ratios corresponding to the preferential degree of the BAp $c$-axis are plotted as a function of the bone position in Fig. 9 with standard deviations. Crystallites with the BAp $c$-axis oriented horizontally contribute to the equatorial sections of the diffraction pattern, while crystallites with the $c$-axis oriented vertically contribute to the meridional sections of the diffraction pattern. The meridional regions of the diffraction corresponding to the longitudinal bone axis show a strong dependence to the distribution of the BAp $c$-axis within the bone. In contrast, only weak dependence appears in the equatorial regions.

Both the control and op/op mice exhibit the same pattern as the maximum ratio of $(002) /(310)$ in the close center of the femoral diaphysis. The op/op mouse shows, however, much lower preferential alignment of the BAp $c$-axis at all 


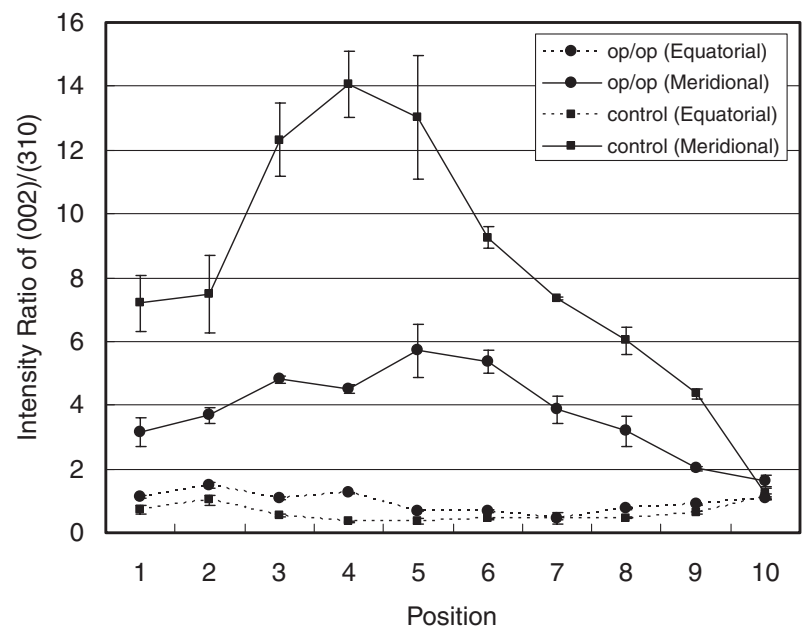

Fig. 9 Distribution of (002)/(310) integrated intensity ratio along longitudinal direction in op/op and control mice. Preferential degrees of BAp $c$ axis as intensity ratios parallel and perpendicular to longitudinal bone axis analyzed at corresponding positions of equatorial and meridional, respectively, as described in Fig. 3.

positions on the femoral position along the longitudinal axis than the control mouse. A similar tendency was previously demonstrated only at the center of the femur in the op/op mouse, ${ }^{12,13)}$ but continuous analysis of the BAp orientation was not performed along the longitudinal bone axis because sectioning bone specimens was needed for BAp analysis. ${ }^{8,12,13)}$ In contrast, the transmission optics system using Mo $\mathrm{K} \alpha \mathrm{X}$-ray radiation with low X-ray absorption enabled analysis of the BAp orientation in the mouse bone without sectioning. Thus, the optics system in this study provides a fuss-free method for analyzing BAp orientation as a bone quality parameter.

The different distribution of BAp orientation between the op/op and control mice, especially along the longitudinal bone axis, may be related to the contribution of osteoclast on the ossification process such as intermembranous and endochondoral ossifications. Detailed analysis is now in progress and will be reported in a separate paper.

\section{Conclusion}

We showed that Mo $\mathrm{K} \alpha \mathrm{X}$-ray radiation is a better choice for data collection to determine the preferred orientation of the BAp $c$-axis in bone specimens due to reduced absorption. We also showed that reduced angular resolution is inconsequential because the (002) and (310) reflections are adequately resolved with Mo $\mathrm{K} \alpha \mathrm{X}$-ray radiation. Finally, there is a clear difference in the integrated ratio of (002)/ (310) depending on the quality of the bone being analyzed.

Our proposed method has several advantages. BAp $c$-axis distribution can be obtained along the longitudinal direction of the bone without sectioning it. This work enables easy analysis of $c$-axis distribution with little specimen preparation. We proved the usefulness of this method using an osteopetrotic (op/op) mouse. Through BAp $c$-axis distribution as a bone quality parameter in the near future, this method (Rigaku R-AXIS BQ system) can be used to understand the following: mechanism of bone growth, diagnoses of diseased bone, regenerated bone process, etc.

\section{Acknowledgements}

KS wishes to thank Yukinori Hara of the Rigaku Corporation for the measurements of the micro-CT data and Youji Moritani of Rigaku Corporation for help with the $\mathrm{X}$-ray diffraction measurements. This study was supported by Priority Assistance for the Formation of Worldwide Renowned Centers of Research-The Global COE Program (Project: Center of Excellence for Advanced Structural and Functional Materials Design) and a Grant-in-Aid for Scientific Research and Development from the Ministry of Education, Culture, Sports, Science and Technology of Japan. This work was partly supported by the Industrial Technology Research Program (Project: 03A47002) in 2003 from the New Energy and Industrial Technology Development Organization (NEDO) of Japan.

\section{REFERENCES}

1) A. Shibata, K. Sasaki and T. Kinefuchi: Advances in X-ray Analysis 35 (1992) 407-413.

2) K. Sasaki, Y. Hirose and T. Sasaki: Advances in X-ray Analysis 37 (1994) 483-490.

3) W. J. Landis: Bone 16 (1995) 533-544.

4) A. Boskey: Osteoporos. Int. 14 (2003) S16-S21.

5) S. Weiner and H. D. Wagner: Annu. Rev. Mater. Sci. 28 (1998) 271-298.

6) R. Marcus, M. Wong, H. Heath III and J. L. Stock: Endocr. Rev. 23 (2002) 16-37.

7) J. F. Veenland, T. M. Link, W. Konermann, N. Meier, J. L. Grashuis and E. S. Gelsema: Calcif. Tissue Int. 61 (1997) 474-479.

8) T. Nakano, Y. Kaibara, Y. Tabata, N. Nagata, S. Enomoto, E. Marukawa and Y. Umakoshi: Bone 31 (2002) 479-487.

9) T. Nakano, Y. Kaibara, Y. Tabata, N. Nagata, S. Enomoto, E. Marukawa and Y. Umakoshi: Tissue engineering for therapeutic use 6, (Analysis of hydroxyapatite (HAp) texture in regenerated hard tissues using micro-beam X-ray diffractmeter technique, Elsevier, Tokyo 2002) 95-104.

10) T. Nakano, T. Ishimoto, Y. Umakoshi and Y. Tabata: Mater. Sci. Forum 595-543 (2007) 675-680.

11) N. Sasaki, N. Matsushima, T. Ikawa, J. Yamamura and A. Fukuda: J. Biomech. 22 (1989) 157-164.

12) J.-W. Lee, T. Nakano, S. Toyosawa, N. Ijuhin, Y. Tabata, M. Yamamoto and Y. Umakoshi: Mater. Sci. Forum 512 (2006) 265-268.

13) J.-W. Lee, T. Nakano, S. Toyosawa, Y. Tabata and Y. Umakoshi: Mater. Trans. 48 (2007) 337-342. 